\title{
Proteomic analysis of lymphoblastoid cell lines from schizophrenic patients
}

\author{
Akira Yoshimi $\mathbb{1}^{1,2,3}$, Shinnosuke Yamada ${ }^{1,2}$, Shohko Kunimoto ${ }^{3}$, Branko Aleksic $\mathbb{B}^{3}$, Akihiro Hirakawa ${ }^{4}$, Mitsuki Ohashi ${ }^{1}$, \\ Yurie Matsumoto ${ }^{1,3}$, Kazuhiro Hada2, Norimichi Itoh2, Yuko Arioka ${ }^{3,5}$, Hiroki Kimura ${ }^{3,6}$, Itaru Kushima 3,6,7, \\ Yukako Nakamura ${ }^{3}$, Tomoko Shiino $\mathbb{B}^{3,8}$, Daisuke Mori $\mathbb{1}^{3,9}$, Satoshi Tanaka $\mathbb{C D}^{6}$, Shuko Hamada ${ }^{3}$, Yukihiro Noda ${ }^{1,2,3}$, \\ Taku Nagai ${ }^{2}$, Kiyofumi Yamada ${ }^{2}$ and Norio Ozaki $\mathbb{1}^{3,6}$
}

\begin{abstract}
Although a number of studies have identified several convincing candidate genes or molecules, the pathophysiology of schizophrenia (SCZ) has not been completely elucidated. Therapeutic optimization based on pathophysiology should be performed as early as possible to improve functional outcomes and prognosis; to detect useful biomarkers for SCZ, which reflect pathophysiology and can be utilized for timely diagnosis and effective therapy. To explore biomarkers for SCZ, we employed fluorescence two-dimensional differential gel electrophoresis (2D-DIGE) of lymphoblastoid cell lines (LCLs) [1st sample set: 30 SCZ and 30 control subjects (CON)]. Differentially expressed protein spots were sequenced by liquid chromatography tandem-mass spectrometry (LC-MS/MS) and identified proteins were confirmed by western blotting (WB) (1st and 2nd sample set: 60 SCZ and 60 CON). Multivariate logistic regression analysis was performed to identify an optimal combination of biomarkers to create a prediction model for SCZ. Twenty protein spots were differentially expressed between SCZ and CON in 2D-DIGE analysis and 22 unique proteins were identified by LC-MS/MS. Differential expression of eight of 22 proteins was confirmed by WB. Among the eight candidate proteins (HSPA4L, MX1, GLRX3, UROD, MAPRE1, TBCB, IGHM, and GART), we successfully constructed logistic regression models comprised of 4- and 6-markers with good discriminative ability between SCZ and CON. In both WB and gene expression analysis of $L C L, M X 1$ showed reproducibly significant associations. Moreover, $M \times 1$ and its related proinflamatory genes $(M \times 2, \| 16$, and Tnf) were also up-regulated in poly I:C-treated mice. Differentially expressed proteins might be associated with molecular pathophysiology of SCZ, including dysregulation of immunological reactions and potentially provide diagnostic and prognostic biomarkers.
\end{abstract}

\section{Introduction}

Schizophrenia (SCZ) is a chronic and disabling mental disorder, typically occurring after puberty, with a lifetime prevalence of $\sim 1 \%$ in the global population. Symptoms of SCZ are characterized by various clinical features including positive (psychosis, hallucinations, delusions,

\footnotetext{
Correspondence: Branko Aleksic (branko@med.nagoya-u.ac.jp)

${ }^{1}$ Division of Clinical Sciences and Neuropsychopharmacology, Faculty and Graduate School of Pharmacy, Meijo University, Nagoya 468-8503, Japan

${ }^{2}$ Department of Neuropsychopharmacology and Hospital Pharmacy, Nagoya University Graduate School of Medicine, Nagoya 466-8550, Japan

Full list of author information is available at the end of the article.

These authors contributed equally: Akira Yoshimi, Shinnosuke Yamada, Shohko Kunimoto
}

and disorganized speech/thinking/behaviour) and negative (apathy, lack of emotion, and social withdrawal) symptoms, as well as cognitive deficits (difficulties in working and long-term memory, attention, and executive function), which cause enormous personal and societal burdens ${ }^{1}$. The duration of untreated psychosis has been reported as one of the most relevant clinical response predictors for SCZ. Untreated psychosis results in changes in brain structure and function due to neurotoxic effects, and treatment delays worsen clinical and social outcomes ${ }^{2}$. However, since there is no objective biomarker for SCZ, it is difficult to provide more precise early-stage detection. Therefore, reliable biomarkers

\section{(c) The Author(s) 2019}

(c) (i) Open Access This article is licensed under a Creative Commons Attribution 4.0 International License, which permits use, sharing, adaptation, distribution and reproduction c. in any medium or format, as long as you give appropriate credit to the original author(s) and the source, provide a link to the Creative Commons license, and indicate if changes were made. The images or other third party material in this article are included in the article's Creative Commons license, unless indicated otherwise in a credit line to the material. If material is not included in the article's Creative Commons license and your intended use is not permitted by statutory regulation or exceeds the permitted use, you will need to obtain permission directly from the copyright holder. To view a copy of this license, visit http://creativecommons.org/licenses/by/4.0/. 
based on the pathophysiology of SCZ have been desired and could point towards new therapeutic/preventive strategies $^{3}$.

SCZ is a disease of the central nervous system (CNS) and investigations of brain samples will no doubt provide meaningful information, however it is very difficult to obtain brain biopsy samples from patients. Although post-mortem brains have been investigated, these samples are affected by various confounding factors related to the age at death, medication, cause of death, agonal state, post-mortem interval, and brain $\mathrm{pH}^{4}$. Several studies have suggested that CNS alterations might be reflected in peripheral tissues based on gene expression profiles between brain and blood ${ }^{5}$. Although fresh blood is useful for screening peripheral biomarkers, it is also affected by various confounding factors such as health condition, diet, medication, smoking, and circadian rhythms ${ }^{3}$. In contrast, the use of cell-lines with repeated passaging could reduce the effect of the above mentioned confounding factors and is useful to identify molecules underlying pathophysiology ${ }^{6}$. Proteomic approaches, as well as microarray or next generation sequencing (NGS)-based transcriptome studies, have been demonstrated to be particularly useful for screening molecular expression changes to obtain novel insights into disease ${ }^{7}$. In particular, proteomic analyses have many advantages related to the major role of proteins in molecular function, thereby reflecting disease state and/or traits. Therefore, proteomics could provide molecular biology based diagnostic tools (biomarkers) for monitoring symptom severity, treatment responses, and predicting progression.

To explore differentially expressed proteins and potential biomarkers for SCZ, we conducted twodimensional fluorescence difference gel electrophoresis (2D-DIGE) analyses of lymphoblastoid cell lines (LCLs).

\section{Materials and methods \\ Subjects}

Two independent sample groups were used in this study (Supplementary Table S1). All subjects were unrelated to each other and ethnically Japanese. The SCZ diagnosis was made by at least two experienced psychiatrists and was based on unstructured patient interviews and reviews of their medical records in accordance with the Diagnostic and Statistical Manual of Mental Disorders, Fourth Edition, Text Revision (DSM-IV-TR) criteria. All control subjects $(\mathrm{CON})$ were selected from the general population and psychiatrically screened by interviews. This study was approved by the Ethics Review Committee of Nagoya University Graduate School of Medicine and Nagoya University Hospital (No. 1033), and was conducted in accordance with the Helsinki Declaration. Written informed consent was obtained from each subject.

\section{Cell cultures}

LCLs derived from SCZ $(n=60)$ and CON $(n=60)$ subjects were established with the widely-used EpsteinBarr virus (EBV) transformation ${ }^{8}$, with minor modifications. In brief, $5 \mathrm{~mL}$ of venous blood was drawn into a vacuum blood collection tube with sodium heparin, and lymphocytes ware isolated using Ficoll-Paque (Amersham Biosciences, Piscataway, NJ, USA) density gradient centrifugation. After washing the lymphocytes with saline, cells were cultured in Roswell Park Memorial Institute (RPMI) 1640 medium with $2 \mathrm{mM} \mathrm{L}$-glutamine and $5 \mathrm{mg} / \mathrm{L}$ phenol red (Gibco, Big Cabin, OK, USA), supplemented with $20 \%$ heat-inactivated foetal bovine serum (FBS, single lot, as was media to minimize variation; Gibco), $8 \mathrm{mg} / \mathrm{L}$ tylosin tartrate (Sigma-Aldrich, St. Louis, MO, USA), $50 \mathrm{U} / \mathrm{mL}$ penicillin $-50 \mu \mathrm{g} / \mathrm{mL}$ streptomycin (Gibco), filtered supernatant of B95-8 cell cultures infected by EBV (VR-1492; American Type Culture Collection, Rockville, MD, USA), and $2 \mu \mathrm{g} / \mathrm{mL}$ cyclosporine A (CyA) (Sandimmune; Novartis Pharma, Tokyo, Japan) under optimal growth conditions at $37^{\circ} \mathrm{C}$ in a humidified $5 \% \mathrm{CO}_{2}$ incubator. Cells were passaged twice a week using RPMI1640 medium (supplemented with $10-20 \%$ heat-inactivated FBS, $8 \mu \mathrm{g} / \mathrm{mL}$ tyrosine tartrate, $50 \mathrm{U} / \mathrm{mL}$ penicillin $-50 \mu \mathrm{g} /$ $\mathrm{mL}$ streptomycin without EBV and CyA). After colony formation, cells were pelleted and stored in liquid nitrogen until analyses. For expression analyses, stored LCLs were re-cultured by rapidly thawing at $37^{\circ} \mathrm{C}$ and were grown in RPMI 1640 medium at $37{ }^{\circ} \mathrm{C}$ with $5 \% \mathrm{CO}_{2}$. Cell growth was monitored with a haemocytometer, and cells were cultured at a density of $\sim 3.0 \times 10^{5}$ cells $/ \mathrm{mL}$ by exchanging the medium with fresh media twice a week. To avoid the cause of altered cellular function (e.g. enhancement of endoplasmic reticulum stress response) ${ }^{9}$, we used LCLs with the number of freeze and thaw only at once. In addition, to minimize differences in expression profiles due to culture and sample workup conditions, all samples were cultured and harvested at the same time using the same media preparation and extraction reagents.

\section{D-DIGE analysis}

To explore differentially expressed protein profiles between SCZ and CON, LCLs from the 1st sample set (SCZ $n=30, \operatorname{CON} n=30$ ) were homogenized in lysis buffer (20 mM Tris- $\mathrm{HCl}$, pH 7.5, $2 \mathrm{mM}$ EDTA, $0.5 \mathrm{mM}$ EGTA, $1 \mathrm{mM}$ dithiothreitol (DTT), 1\% Triton X-100, protease inhibitor cocktail, phosphatase inhibitor cocktail) and centrifuged at $16000 \times g$ for $20 \mathrm{~min}$ at $4{ }^{\circ} \mathrm{C}$. The soluble supernatant was purified and concentrated with methanol/acetone precipitation and reconstituted in resuspension buffer (30 mM Tris- $\mathrm{HCl}, \mathrm{pH}$ 8.5, 4\% CHAPS, $7 \mathrm{M}$ urea, $2 \mathrm{M}$ thiourea). Protein concentrations were determined using a commercial protein assay kit (Bio-Rad, Hercules, CA, USA). 
2D-DIGE was carried out according to a previously published method ${ }^{10}$ with minor modifications. An equal amount of protein $(30 \mu \mathrm{g})$ from each LCL was individually labelled using $240 \mathrm{pmol}$ of either Cy3 or Cy5 from a CyDye DIGE Fluor Minimal Labelling Kit (GE Healthcare, Chalfont St. Giles, Buckinghamshire, UK), according to the manufacturer's protocol. For spot normalization to allow comparison across different gels, we prepared an internal standard (IS) protein pool consisting of equal amounts of all samples, which was labelled with Cy2. Fluorescently labelled proteins were diluted with an equal volume of sample buffer [ $40 \mathrm{mM}$ DTT, $4 \%$ CHAPS, $7 \mathrm{M}$ urea, $2 \mathrm{M}$ thiourea, $1 \%$ pharmalyte (broad range pH 3-10)]. Different kinds of fluorescent-labelled LCL protein samples from SCZ, CON, and IS were mixed before loading on the gel. Mixed samples were added to a final volume $(450 \mu \mathrm{L})$ of rehydration buffer [20 mM DTT, $4 \%$ CHAPS, $7 \mathrm{M}$ urea, $2 \mathrm{M}$ thiourea, $0.5 \%$ pharmalyte, $0.001 \%$ bromophenol blue (BPB)] and were applied to IPG gel strips with a separation range of pH 3-10 (24 cm Immobiline DryStrip pH 3-10 $\mathrm{NL}, 240 \times 3 \times 0.5 \mathrm{~mm}$; GE Healthcare). After $12 \mathrm{~h}$ of rehydration at $20^{\circ} \mathrm{C}$, isoelectric focusing (IEF) was carried out as follows; initially at $30 \mathrm{~V}$ for $2 \mathrm{~h}$, at $100 \mathrm{~V}$ for $1 \mathrm{~h}$, at $200 \mathrm{~V}$ for $5 \mathrm{~min}$, and then gradually increasing the voltage to $8000 \mathrm{~V}$ for $8.5 \mathrm{~h}$, and finally maintaining at $8000 \mathrm{~V}$ until reaching 60,000 Vh in an Ettan IPGphor 3 Isoelectric Focusing System (GE Healthcare), maintaining a limiting current of $50 \mu \mathrm{A}$ per strip at $20^{\circ} \mathrm{C}$.

After IEF separation, the drystrip gels were equilibrated for $25 \mathrm{~min}$ in sodium dodecyl sulphate (SDS)-equilibration buffer $(50 \mathrm{mM}$ Tris- $\mathrm{HCl}, \mathrm{pH} 8.8,6 \mathrm{M}$ urea, $30 \%$ glycerol, 2\% SDS) with 1\% DTT for reduction. Equilibration was repeated in the SDS-equilibration buffer for another $10 \mathrm{~min}$ with $2.5 \%$ iodoacetamide for alkylation. The second-dimensional separation was carried out on a $10 \%$ SDS-polyacrylamide gel $(24 \times 20 \times 0.1 \mathrm{~cm})$. SDSpolyacrylamide gel electrophoresis (SDS-PAGE) was performed using a two-step protocol; at $30^{\circ} \mathrm{C}, 10 \mathrm{~mA} / \mathrm{gel}$, $80 \mathrm{~V}, 1 \mathrm{~W} /$ gel for an hour, and then $12 \mathrm{~mA} / \mathrm{gel}, 150 \mathrm{~V}$, $2 \mathrm{~W} /$ gel for $15-17 \mathrm{~h}$ until the loading marker reached the edge of the gel in the Ettan DALTsix Large Electrophoresis System (GE Healthcare).

Fluorescence dye (Cy2, Cy3, and Cy5) labelled proteins were visualized by scanning gels at $100 \mu \mathrm{m}$ resolution using a Typhoon Trio laser scanner (GE Healthcare). A total of 87 images from 29 gels with good separation quality were analysed using PDQuest 2-D Analysis Advanced Software Version 8.0 (Bio-Rad). The abundance of each Cy3- or Cy5labeled protein spot was normalized according to the corresponding protein spot from the Cy2-labeled IS sample.

\section{Mass spectrometry}

For protein identification, $100 \mu \mathrm{g}$ of the un-labelled IS protein pool was separated by 2D-PAGE as described above. The proteins were visualized by silver staining and the protein spots of interest were excised. The gel pieces including proteins were destained in destaining solution $\left(0.2 \% \mathrm{~K}_{3} \mathrm{Fe}(\mathrm{CN})_{6}\right.$ and $\left.0.02 \% \mathrm{Na}_{2} \mathrm{~S}_{2} \mathrm{O}_{3}\right)$ for $15 \mathrm{~min}$, washed in Milli-Q water for $10 \mathrm{~min}$, dehydrated in $\mathrm{CH}_{3} \mathrm{CN}$, and dried in a centrifuge-vacuum dryer (Spin Dryer Lite VC36R; TAITEC) for $15 \mathrm{~min}$. The gels were rehydrated and reduced with $100 \mathrm{mM}$ DTT in $100 \mathrm{mM} \mathrm{NH}_{4} \mathrm{HCO}_{3}$ for $30 \mathrm{~min}$ at $50^{\circ} \mathrm{C}$, and then dehydrated as described above. The gel pieces were alkylated with $100 \mathrm{mM}$ iodoacetamide in $100 \mathrm{mM} \mathrm{NH} \mathrm{NCO}_{3}$ for $30 \mathrm{~min}$ at room temperature (RT), dehydrated, and rehydrated by $20 \mu \mathrm{g} / \mathrm{mL}$ trypsin (Promega Benelux, Leiden, The Netherlands) in $100 \mathrm{mM}$ $\mathrm{NH}_{4} \mathrm{HCO}_{3}$ for $30 \mathrm{~min}$ on ice. A solution of $100 \mathrm{mM}$ $\mathrm{NH}_{4} \mathrm{HCO}_{3}(10-15 \mu \mathrm{L})$ was added, and the proteins were digested overnight at $37^{\circ} \mathrm{C}$. Peptides were extracted three times with $50 \mu \mathrm{L}$ of Solution A $\left(2 \% \mathrm{CH}_{3} \mathrm{CN}\right.$ and $0.1 \%$ TFA), $33 \mu \mathrm{L}$ of Solution B ( $98 \% \mathrm{CH}_{3} \mathrm{CN}$ and $0.1 \%$ TFA), and $42 \mu \mathrm{L}$ of Solution B. The extracts were dried and dissolved in Solution A (total 10-12 $\mu \mathrm{L}$ ) prior to application to the sample vial. Each sample of peptides was loaded on a Paradigm MS4 HPLC system (Michrom BioResources, Auburn, CA, USA) equipped with a magic C18AQ column of $0.1 \mathrm{~mm}$ in diameter and $50 \mathrm{~mm}$ in length (Michrom BioResources). Reversed-phase chromatography was performed with a linear gradient of solvent $\mathrm{A}\left(2 \% \mathrm{CH}_{3} \mathrm{CN}\right.$ in $\left.0.1 \% \mathrm{HCOOH}\right)$ and solvent $\mathrm{B}(90 \%$ $\mathrm{CH}_{3} \mathrm{CN}$ in $0.1 \% \mathrm{HCOOH}$ ), $5 \%$ solvent $\mathrm{B}$ at 0 min to $100 \%$ solvent $\mathrm{B}$ at $45 \mathrm{~min}$ with a $1 \mu \mathrm{L} / \mathrm{min}$ flow rate. Ionization was performed using an ADVANCE CaptiveSpray Source (Michrom BioResources) with a capillary voltage of $1.7 \mathrm{kV}$ and temperature of $150^{\circ} \mathrm{C}$. A precursor ion scan was carried out using a 400-2000 mass to charge ratio $(\mathrm{m} / \mathrm{z})$ prior to MS/MS analysis with an LCQ advantage mass spectrometer (Thermo Fisher Scientific, Waltham, MA, USA). Multiple peptide mass fingerprinting and MS/MS peptide fragmentation data were used for searching the Swiss-Prot protein database with the MASCOT program (Matrix Science, Boston, MA, USA) for MS/MS ion searching with $2.0 \mathrm{D}$ mass tolerance. Identified proteins were classified according to functional ontology using the Human Protein Reference Database (HPRD: http://www. hprd.org).

\section{Western blotting}

Equal amounts $(15 \mu \mathrm{g})$ of proteins extracted from LCLs of the 1st (SCZ $n=30, \operatorname{CON} n=30)$ and 2nd (SCZ $n=$ 30 , $\mathrm{CON} n=30$ ) sample sets (see above procedure) were diluted in sample buffer (final concentration: $62.5 \mathrm{mM}$ Tris- $\mathrm{HCl}, \mathrm{pH} 6.8$, containing $2 \%$ SDS, $10 \%$ glycerol, $0.5 \%$ $\mathrm{BPB}$, and 5\% 2-mercaptoethanol) and denatured at $95^{\circ} \mathrm{C}$ for $5 \mathrm{~min}$. Proteins were separated by SDS-PAGE using $5 \%$ stacking gels and $8-12 \%$ running gels and transferred to PVDF membranes using a Trans-Blot SD Semi-Dry 
Transfer Cell (Bio-Rad). Membrane blots were processed using the SNAP i.d. protein detection system (Millipore), according to the manufacturer's protocol, or placing the blots into a Hybri-Bag at RT for $1 \mathrm{~h}$ or overnight at $4{ }^{\circ} \mathrm{C}$, with specific antibodies or horseradish peroxidaseconjugated secondary antibodies (Supplementary Table S2). The positive bands were revealed using enhanced chemiluminescence substrate for western blotting (WB) reagents (PerkinElmer, Waltham, MA, USA) and visualized with an ImageQuant LAS 4000mini (GE Healthcare). The intensities of the bands were quantified using ImageQuant TL 7.0 software (GE Healthcare).

\section{Animals}

ICR mice were obtained from Japan SLC Inc. (Hamamatsu, Japan) and maintained under standard specific pathogen-free environmental conditions. Pregnant mice were monitored for the parturition date, which was taken as postnatal day (PD) 0 . They were housed under a standard 12-h light/dark cycle (lights on at 9:00) at a constant temperature of $23 \pm 1{ }^{\circ} \mathrm{C}$, with free access to food and water throughout the experiments. We used male mice exclusively to minimize any potential variability due to sexspecific effects. The animals were handled in accordance with the guidelines established by the Institutional Animal Care and Use Committee of Nagoya University Graduate School of Medicine (approval ID: 30316, 30416) and Meijo University Faculty of Pharmacy (approval ID: 2018-P-E-16), the Guiding Principles for the Care and Use of Laboratory Animals approved by the Japanese Pharmacological Society, and the National Institutes of Health Guide for the Care and Use of Laboratory Animals (NIH Publications No. 8023, revised 1978).

\section{Gene expression analysis of prefrontal cortex and hippocampus of poly I:C-treated mice}

All litters were randomly divided into saline- or poly I: C-treated groups. From PD 2 to 6, mice were injected s.c. daily with either pyrogen-free saline or poly I:C (Sigma-Aldrich) at a dose of $5 \mathrm{mg} / \mathrm{kg}^{11}$. Neonatal mice were decapitated 2 or $24 \mathrm{~h}$ after the final treatment with saline or poly I:C and their brains were removed. Total RNA was isolated using the RNeasy Plus Mini Kit (Qiagen, Hilden, Germany) from each mouse ( $2 \mathrm{~h}$ after the final treatment with saline $n=6$ or poly I:C $n=6,24 \mathrm{~h}$ after the final treatment with saline $n=6$ or poly I:C $n=$ 6). Total RNA isolated from the prefrontal cortex (PFC) and hippocampus (HIP) was converted into cDNA using a high capacity RNA-to-cDNA Kit (Applied Biosystems, Foster City, CA, USA). Levels of mRNA expression were quantified using an ABI Prism 7900HT Real-Time PCR System (Applied Biosystems) with the KAPA SYBR ${ }^{\circ}$ FAST qPCR Kit (Kapa Biosystems, Boston, MA, USA), according to the manufacturer's directions. The primer pairs for eight candidate markers confirmed by WB (Table 2), proinflammatory mediators (Myxovirus resistance (Mx) 2: MxI; Interferon $\alpha$ : Ifna; Interferon $\beta$ : Ifnb; Interferon $\gamma$ : Ifng; Interleukin $1 \beta$ : Il1b; Interleukin 6: Il6; Tumor necrosis factor $\alpha$ : Tnf; Nuclear factor of kappa light polypeptide gene enhancer in B cells 1, p105: $N f k b$ ), and a housekeeping gene ( $\beta$-actin: $A c t b)$ are described in Supplementary Table S3.

\section{Statistical analysis}

Sample sizes were determined based on literature ${ }^{10,12}$ and were not calculated using statistical methods. Data collection and analysis were not blind to the sample conditions. Data were analyzed using either Student's $t$-test or Welch's $t$-test for comparisons between two groups. The comparison of expression levels of identified candidate markers was performed using univariate logistic regression analysis. To search optimal prediction models for SCZ, multivariate logistic regression approach was performed. $P$ values less than 0.05 were considered to be statistically significant.

\section{Results}

Altered protein expression in LCLs between SCZ and CON

A representative merged 2D-DIGE image of LCL from SCZ and CON subjects is shown in Supplementary Figure S1. A total of 1174 protein spots were identified on typical LCL gels [SCZ 1102.8 (s.d. = 13.3); CON 1102.6 $($ s.d. = 16.2)], with 20 spots being differentially expressed between SCZ-LCL and CON-LCL, of which seven spots were increased while 13 spots were decreased in SCZ-LCL (Table 1). These 20 differentially expressed protein spots were selected for protein identification by liquid chromatography tandem-mass spectrometry (LC-MS/MS) and 22 unique proteins were successfully identified with high MASCOT scores (Table 1).

\section{Western blotting validation}

WB analysis of the 22 identified proteins was performed to confirm the results of the 2D-DIGE analysis in 1st sample set (SCZ $n=30, \operatorname{CON} n=30$ ). The direction of change was confirmed in eight of the 22 proteins (Table 2, Supplementary Figures S2 and S3). MX1 and IGHM were significantly up-regulated in SCZ-LCL, while HSPA4L, GLRX3, UROD, MAPRE1, TBCB, and GART were significantly down-regulated in the 1st sample set. These eight proteins were examined for further validation analysis using an independent 2nd sample set ( $\operatorname{SCZ} n=30, \operatorname{CON} n=30)$. As a result, three proteins (HSPA4L, MX1, and GART) were found to be differentially expressed in the 2nd sample set with the same direction of change (Table 2 and Supplementary Figure S4). When we combined the results of the two sample sets, four proteins (HSPA4L, MX1, TBCB, and GART) were found to be significantly altered (Table 2 and Supplementary Figure S5). 


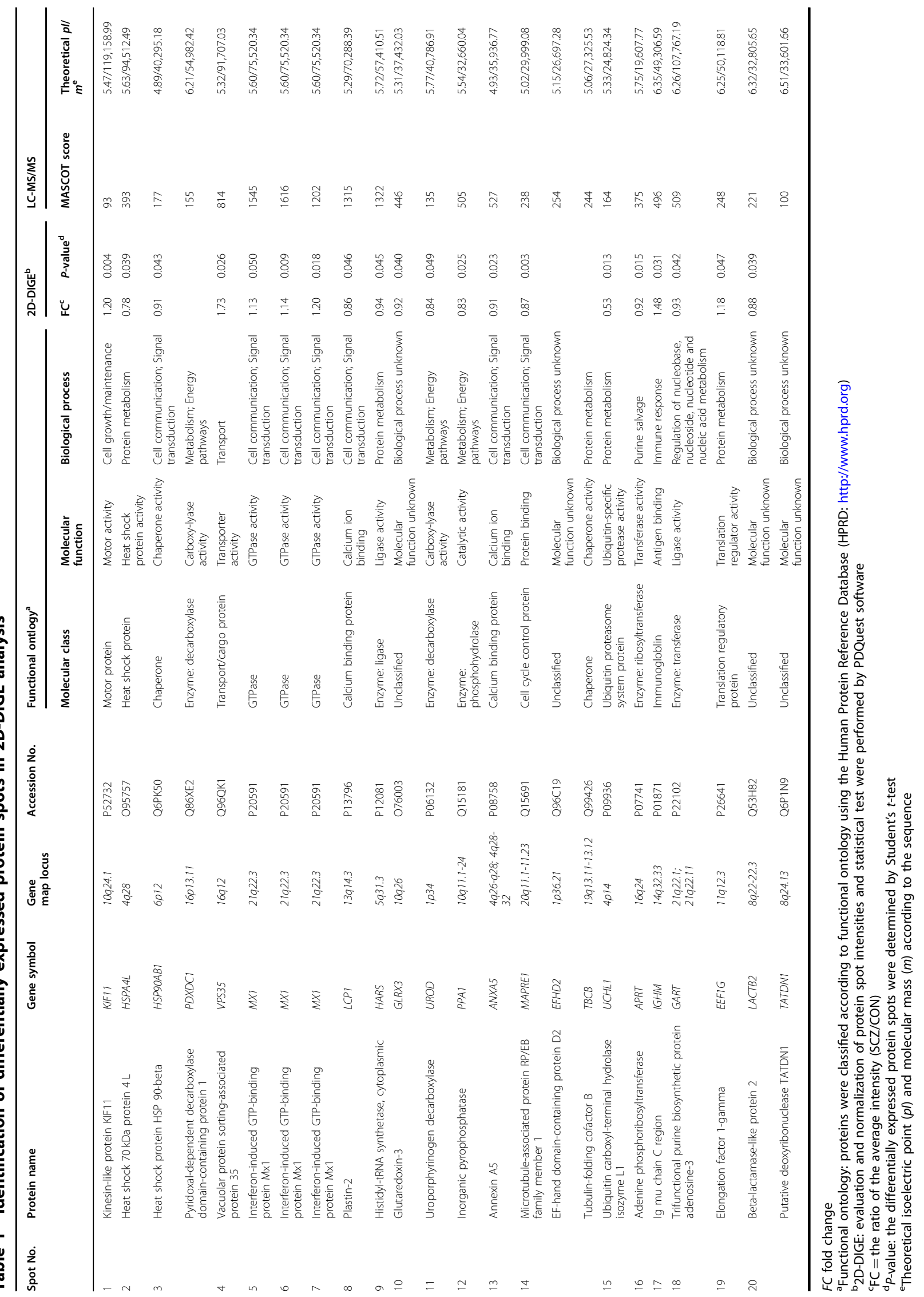


Table 2 Results of Western blotting analysis of LCLs

\begin{tabular}{|c|c|c|c|c|c|c|c|c|c|c|c|c|c|c|c|c|c|c|c|}
\hline \multirow[t]{3}{*}{ Spot No. } & \multirow[t]{3}{*}{ Candidate marker } & \multicolumn{6}{|c|}{$\begin{array}{l}\text { 1st sample set (CON } n=30, \mathrm{SCZ} \\
n=30)\end{array}$} & \multicolumn{6}{|c|}{$\begin{array}{l}\text { 2nd sample set (CON } n=30, \text { SCZ } \\
n=30)\end{array}$} & \multicolumn{6}{|c|}{ Combinede (CON $n=60, \operatorname{SCZ} n=60)$} \\
\hline & & \multirow[t]{2}{*}{$\mathrm{FC}^{\mathrm{a}}$} & \multirow{2}{*}{\multicolumn{2}{|c|}{$\begin{array}{l}t \text {-test } P \text { - } \\
\text { value }^{b}\end{array}$}} & \multicolumn{3}{|c|}{$\begin{array}{l}\text { Logistic } \\
\text { regression } \\
\text { analysis }\end{array}$} & \multirow[t]{2}{*}{ FC } & \multicolumn{2}{|c|}{$\begin{array}{l}t \text {-test } P \text { - } \\
\text { value }\end{array}$} & \multicolumn{3}{|c|}{$\begin{array}{l}\text { Logistic regression } \\
\text { analysis }\end{array}$} & \multirow[t]{2}{*}{$\mathrm{FC}$} & \multirow{2}{*}{\multicolumn{2}{|c|}{$\begin{array}{l}t \text {-test } P \text { - } \\
\text { value }\end{array}$}} & \multicolumn{3}{|c|}{$\begin{array}{l}\text { Logistic regression } \\
\text { analysis }\end{array}$} \\
\hline & & & & & OR & $P$-value & AUC & & & & OR & $P$-value & AUC & & & & OR & $P$-value & AUC \\
\hline 1 & KIF11 & 1.31 & 0.284 & (S) & 2.10 & 0.289 & 0.63 & & & & & & & & & & & & \\
\hline 2 & HSPA4L & 0.62 & 0.017 & $(W)$ & 0.50 & 0.029 & 0.64 & 0.73 & 0.015 & $(\mathrm{~W})$ & 0.05 & 0.021 & 0.65 & 0.65 & $<0.001$ & $(\mathrm{~W})$ & 0.59 & 0.03 & 0.61 \\
\hline \multirow[t]{2}{*}{3} & HSP90AB1 & 1.12 & 0.390 & $(\mathrm{~S})$ & 1.45 & 0.384 & 0.57 & & & & & & & & & & & & \\
\hline & PDXDC1 & 1.10 & 0.604 & (S) & 1.15 & 0.598 & 0.49 & & & & & & & & & & & & \\
\hline 4 & VPS35 & 0.88 & 0.295 & (S) & 0.39 & 0.296 & 0.55 & & & & & & & & & & & & \\
\hline $5,6,7$ & MX1 & 1.39 & 0.006 & $(\mathrm{~S})$ & 9.26 & 0.011 & 0.72 & 1.32 & 0.006 & $(W)$ & 12.76 & 0.012 & 0.68 & 1.35 & $<0.001$ & $(W)$ & 10.32 & $<0.001$ & 0.70 \\
\hline 8 & LCP1 & 1.04 & 0.791 & (S) & 1.07 & 0.787 & 0.54 & & & & & & & & & & & & \\
\hline 9 & HARS & 1.09 & 0.588 & (S) & 1.55 & 0.582 & 0.57 & & & & & & & & & & & & \\
\hline 10 & GLRX3 & 0.74 & 0.002 & $(W)$ & 0.05 & 0.006 & 0.73 & 1.12 & 0.103 & (S) & 13.31 & 0.106 & 0.61 & 0.88 & 0.073 & (S) & 0.33 & 0.08 & 0.55 \\
\hline 11 & UROD & 0.77 & 0.010 & (S) & 0.24 & 0.018 & 0.73 & 1.03 & 0.824 & $(W)$ & 1.17 & 0.828 & 0.54 & 0.85 & 0.118 & (S) & 0.60 & 0.12 & 0.59 \\
\hline 12 & PPA1 & 0.99 & 0.953 & (S) & 0.97 & 0.952 & 0.50 & & & & & & & & & & & & \\
\hline 13 & ANXA5 & 0.88 & 0.105 & $(W)$ & 0.32 & 0.109 & 0.61 & & & & & & & & & & & & \\
\hline \multirow[t]{3}{*}{14} & MAPRE1 & 0.76 & 0.009 & (S) & 0.20 & 0.015 & 0.69 & 1.18 & 0.010 & (S) & 95.47 & 0.015 & 0.68 & 0.89 & 0.182 & (S) & 0.57 & 0.19 & 0.51 \\
\hline & EFHD2 & 0.99 & 0.908 & (S) & 0.95 & 0.906 & 0.47 & & & & & & & & & & & & \\
\hline & TBCB & 0.68 & 0.024 & $(W)$ & 0.20 & 0.034 & 0.66 & 0.95 & 0.628 & (S) & 0.68 & 0.622 & 0.57 & 0.82 & 0.035 & (S) & 0.36 & 0.04 & 0.60 \\
\hline 15 & UCHL1 & 0.97 & 0.919 & (S) & 0.99 & 0.917 & 0.54 & & & & & & & & & & & & \\
\hline 16 & APRT & 0.59 & 0.248 & $(W)$ & 0.90 & 0.356 & 0.49 & & & & & & & & & & & & \\
\hline 17 & IGHM & 1.80 & 0.037 & $(W)$ & 5.03 & 0.050 & 0.63 & 0.71 & 0.336 & $(W)$ & 0.98 & 0.336 & 0.49 & 0.80 & 0.466 & (S) & 0.98 & 0.46 & 0.45 \\
\hline 18 & GART & 0.75 & 0.022 & (S) & 0.15 & 0.031 & 0.66 & 0.73 & 0.002 & (S) & 0.02 & 0.005 & 0.74 & 0.74 & $<0.001$ & $(\mathrm{~W})$ & 0.08 & $<0.001$ & 0.70 \\
\hline 19 & EEF1G & 1.16 & 0.154 & (S) & 2.35 & 0.158 & 0.65 & & & & & & & & & & & & \\
\hline \multirow[t]{2}{*}{20} & LACTB2 & 1.10 & 0.358 & (S) & 1.76 & 0.356 & 0.56 & & & & & & & & & & & & \\
\hline & TATDN1 & 1.14 & 0.195 & $(W)$ & 2.58 & 0.210 & 0.57 & & & & & & & & & & & & \\
\hline
\end{tabular}

FC fold change, OR odds ratio (SCZ/CON), AUC the area under the receiver operating characteristic curves, Combined combined results of 1 st sample set and 2 nd sample set

${ }^{\mathrm{a}} \mathrm{FC}=$ the ratio of the average intensity (SCZ/CON)

${ }^{b} t$-test $P$-value: the differentially expressed proteins were determined by Student's $t$-test (S) or Welch's $t$-test (W) (unpaired, two tailed)

\section{Statistical prediction model}

Among the eight promising biomarkers validated by WB in the 1st sample set, optimal 4-marker model (comprised of MX1, GLRX3, UROD, and GART; equation 1) and 6-maker model (comprised of MX1, GLRX3, UROD, MAPRE1, TBCB, and GART; equation 2) were constructed by multivariate logistic regression analysis.

$$
p=\frac{\exp (6.44-3.82 G A R T-2.18 U R O D+2.48 M X 1-2.84 G L R X 3)}{1+\exp (6.44-3.82 G A R T-2.18 U R O D+2.48 M X 1-2.84 G L R X 3)}
$$

$p=\frac{\exp (11.54-4.64 G A R T-2.67 U R O D+2.04 M X 1-3.14 G L R X 3-1.65 M A R P E 1-2.01 T B C B)}{1+\exp }$
MX1 and GART showed reproducibly significant associations in both the 4- and 6-marker models (Table 3). The area under the receiver operating characteristic curves (AUCs) for the accuracy of predicting SCZ using the 4- and 6-marker models were 0.86 and 0.88 , respectively (Table 3 and Supplementary Figure S6), implying good discriminative ability. For external validation of these statistical prediction models, the WB results using the 2nd sample set were applied to the 4- and 6-marker models. The AUCs of 4- and 6-marker models were 0.72 and 0.66 , respectively (Table 3 ). The predicting accuracies of two most prominently altered markers, MX1 and 
Table 3 Prediction accuracy of 4- and 6-marker models

\begin{tabular}{|c|c|c|c|c|c|c|c|c|c|}
\hline \multirow[t]{2}{*}{ Variable } & \multicolumn{3}{|c|}{ 1st sample set (CON $n=30, \operatorname{SCZ} n=30)$} & \multicolumn{3}{|c|}{$\begin{array}{l}\text { 2nd sample set (CON } n=30, \text { SCZ } \\
n=30)\end{array}$} & \multicolumn{3}{|c|}{ Combined (CON $n=60, S C Z n=60)$} \\
\hline & OR & $P$-value ${ }^{a}$ & Prediction accuracy ${ }^{\mathbf{b}}$ & OR & $P$-value & Prediction accuracy & OR & $P$-value & Prediction accuracy \\
\hline \multicolumn{10}{|c|}{ 4-marker model } \\
\hline$M \times 1$ & 11.91 & 0.017 & & 26.57 & 0.016 & & 19.46 & $<0.001$ & \\
\hline GLRX3 & 0.06 & 0.036 & $81.7 \%$ & 4.99 & 0.507 & $73.3 \%$ & 0.46 & 0.384 & $77.5 \%$ \\
\hline UROD & 0.11 & 0.011 & $(A \cup C=0.86)$ & 0.55 & 0.609 & $(A \cup C=0.72)$ & 0.44 & 0.108 & $(A \cup C=0.82)$ \\
\hline GART & 0.02 & 0.006 & & 0.01 & 0.006 & & 0.01 & $<0.001$ & \\
\hline \multicolumn{10}{|c|}{ 6-marker model } \\
\hline$M \times 1$ & 7.68 & 0.079 & & 16.72 & 0.049 & & 20.48 & $<0.001$ & \\
\hline GLRX3 & 0.04 & 0.03 & & 2.37 & 0.741 & & 0.48 & 0.431 & \\
\hline UROD & 0.07 & 0.012 & $81.7 \%$ & 0.79 & 0.875 & $78.3 \%$ & 0.51 & 0.235 & $77.5 \%$ \\
\hline GART & 0.01 & 0.011 & $(A \cup C=0.88)$ & 0.003 & 0.008 & $(A \cup C=0.66)$ & 0.01 & $<0.001$ & $(A \cup C=0.82)$ \\
\hline MAPRE1 & 0.19 & 0.095 & & 103.8 & 0.052 & & 0.73 & 0.629 & \\
\hline $\mathrm{TBCB}$ & 0.13 & 0.050 & & 1.93 & 0.616 & & 0.42 & 0.138 & \\
\hline
\end{tabular}

OR odds ratio, Combined combined results of 1 st sample set and 2 nd sample set, AUC the area under the receiver operating characteristic curves

${ }^{a} P$-value: multivariate logistic regression analysis

${ }^{b}$ Prediction accuracy: [1-overall misclassification rate $\left.(\mathrm{OMR})\right] \times 100(\%)$

GART, showed high performance even only applying as a single protein marker $\left(\mathrm{AUC}_{\mathrm{MX} 1}: \quad 0.68-0.72\right.$ and AUC $_{\text {GART }}$ : 0.66-0.74; Table 2).

\section{Gene expression analysis of prefrontal cortex and hippocampus of poly I:C-treated mice}

Since the most promising markers for SCZ identified from LCL experiments were related to the immunological reactions, we analysed the mRNA levels of candidate markers and proinflammatory mediators in PFC and HIP of poly I:C-treated mice, which is an animal neurodevelopmental model of SCZ. Among the genes examined, mRNA levels of $M x 1$ (PFC/HIP), $M x 2$ (PFC/HIP), Il1b (HIP), and $\operatorname{Tnf}(\mathrm{HIP})$ were significantly increased $2 \mathrm{~h}$ after the final treatment of poly I:C, compared to the salinetreated control group (Table 4). At $24 \mathrm{~h}$ after poly I:C treatment, Ighm (PFC/HIP) was increased, while Mapre1 (HIP), Ifnb (HIP), and Il6 (PFC) were decreased in the poly I:C-treated group. Although mRNA level of Gart was not changed in both PFC and HIP at 2 and $24 \mathrm{~h}$ after poly $\mathrm{I}: \mathrm{C}$ treatment (Table 4), repeated poly I:C treatment in neonatal mice are likely cause an inflammatory response in PFC and HIP.

\section{Discussion}

Main findings

In the present study, we conducted a comparative proteomic analysis of LCL proteins derived from SCZ and CON subjects (Supplementary Table S1). Twenty-two unique proteins were identified as differentially expressed markers for SCZ using 2D-DIGE and LC-MS/MS (Table 1). Eight proteins (up-regulated in SCZ-LCL: MX1 and IGHM; down-regulated in SCZ-LCL: HSPA4L, GLRX3, UROD, MAPRE1, TBCB, and GART) were confirmed after WB validation using the 1st sample set (Table 2 and Supplementary Figure S3). External validation confirmed that HSPA4L, MX1, and GART were differentially expressed between SCZ-LCL and CON-LCL with the same direction, while MAPRE1 was not consistent with the 1st sample set (Table 2 and Supplementary Figure S4). Overall, HSPA4L, MX1, TBCB, and GART were significantly altered in the combined analysis of the two sample sets (Table 2 and Supplementary Figure S5), suggesting that these proteins were potentially related to dysfunctional molecular pathways in SCZ. Interestingly, the gene expression level of $M X 1$ in LCL and that of $M x 1$ in the brain (prefrontal cortex and hippocampus) of poly I:C-treated mice (one of the most widely used animal model for schizophrenia ${ }^{11}$ ) were also significantly elevated (Supplementary Table S4 and Table 4); therefore MX1 might be an important molecule for understanding SCZ pathophysiology.

Among the eight candidate proteins (HSPA4L, MX1, GLRX3, UROD, MAPRE1, TBCB, IGHM, and GART) confirmed in the 1st sample set (Table 2 and Supplementary Figure S4), we tried to construct a statistical prediction model for SCZ using multivariate logistic regression analysis, and two combinations of proteins 
Table 4 Results of quantitative real-time PCR analysis of poly I:C-treated mice

\begin{tabular}{|c|c|c|c|c|c|c|c|c|}
\hline \multirow[t]{3}{*}{ Gene symbol } & \multicolumn{4}{|l|}{$2 h^{a}$} & \multicolumn{4}{|c|}{$24 h^{b}$} \\
\hline & \multicolumn{2}{|l|}{ PFC } & \multicolumn{2}{|l|}{ HIP } & \multicolumn{2}{|l|}{ PFC } & \multicolumn{2}{|l|}{ HIP } \\
\hline & $\mathrm{FC}^{\mathrm{c}}$ & $P$-value ${ }^{d}$ & FC & $P$-value & FC & $P$-value & FC & $P$-value \\
\hline \multicolumn{9}{|c|}{ Candidate marker } \\
\hline Hspa4l & 1.39 & 0.176 & 0.63 & 0.292 & 1.07 & 0.425 & 1.00 & 0.994 \\
\hline$M \times 1$ & 2.53 & 0.039 & 1.80 & 0.043 & 1.03 & 0.804 & 0.85 & 0.243 \\
\hline Glrx3 & 1.07 & 0.266 & 0.99 & 0.763 & 1.19 & 0.109 & 1.13 & 0.126 \\
\hline Urod & 0.97 & 0.754 & 0.90 & 0.171 & 1.13 & 0.091 & 1.13 & 0.259 \\
\hline Mapre1 & 1.02 & 0.913 & 0.95 & 0.458 & 1.05 & 0.758 & 0.82 & 0.022 \\
\hline Tbcb & 0.97 & 0.879 & 0.86 & 0.390 & 1.01 & 0.964 & 0.96 & 0.676 \\
\hline Ighm & 1.22 & 0.156 & 1.20 & 0.054 & 1.19 & 0.009 & 1.23 & 0.024 \\
\hline Gart & 1.63 & 0.139 & 1.11 & 0.222 & 0.98 & 0.699 & 0.93 & 0.330 \\
\hline \multicolumn{9}{|c|}{ Proinflammatory mediator } \\
\hline$M \times 2$ & 1.53 & 0.029 & 1.61 & 0.042 & 1.13 & 0.310 & 0.96 & 0.528 \\
\hline Ifna & 1.35 & 0.347 & 1.26 & 0.265 & 0.87 & 0.439 & 0.67 & 0.069 \\
\hline Ifnb & 1.44 & 0.280 & 1.21 & 0.392 & 0.87 & 0.391 & 0.64 & 0.039 \\
\hline Ifng & 1.56 & 0.070 & 1.52 & 0.063 & 1.09 & 0.647 & 1.22 & 0.169 \\
\hline $1 / 16$ & 1.34 & 0.171 & 1.52 & 0.014 & 0.98 & 0.854 & 1.30 & 0.076 \\
\hline 116 & 1.03 & 0.931 & 1.24 & 0.432 & 0.62 & 0.013 & 0.62 & 0.089 \\
\hline $\operatorname{Tnf}$ & 1.60 & 0.108 & 2.49 & 0.005 & 0.74 & 0.189 & 0.72 & 0.116 \\
\hline Nfkb1 & 0.94 & 0.377 & 0.90 & 0.278 & 1.00 & 0.990 & 0.99 & 0.972 \\
\hline
\end{tabular}

PFC prefrontal cortex, HIP hippocampus, FC fold change

${ }^{\mathrm{a}} 2 \mathrm{~h}$ : sacrificed $2 \mathrm{~h}$ after final treatment with saline $(n=6)$ or polyl:C $(n=6)$

${ }^{\mathrm{b}} 24 \mathrm{~h}$ : sacrificed $24 \mathrm{~h}$ after final treatment with saline $(n=6)$ or polyl:C $(n=6)$

${ }^{\mathrm{c}} \mathrm{FC}=$ the ratio of the average intensity (poly I:C/saline)

${ }^{\mathrm{d}} P$-value: the differentially expressed proteins were determined by Student's $t$-test

(4- and 6-marker models) proved suitable for discriminating SCZ and CON with high performance (Table 3 and Supplementary Figure S6). According to Occam's razor, a simpler model is preferred over a more complex model. Increasing the number of variables leads to a more complicated model and may result in over fitting. In order to avoid this problem, the model with fewer variables is considered to be the better statistical model. Consistent with this concept, the prediction accuracy of the two models was found to be equivalent. Therefore, the 4marker model is a good prediction model with better accuracy. Furthermore, statistical model validation using independent samples indicated that MX1 and GART showed reproducibly significant associations in both the 4and 6-marker models (Table 3). These proteins were also strongly associated in the WB validation and also showed high performance for discriminating SCZ and CON in univariate logistic regression analysis (Table 2), suggesting these proteins might be promising biomarkers for SCZ.

\section{Possible mechanisms}

Functional characterization of the identified molecules indicated that they were involved in metabolism, energy pathways, and signal transduction (Table 1). Impairments in these pathways were previously identified as a SCZ trait by genetic studies, gene and protein expression studies, and functional analyses ${ }^{13-15}$, suggesting that the proteins identified in this study may be potentially involved in SCZ pathophysiology.

MX1, the most promising molecular signature for SCZ, is a type I interferon (IFN)-inducible protein that exhibits antiviral activity against a variety of RNA viruses ${ }^{16}$. The reported antiviral activity of MX1 indicates that it accelerates cell death through caspase-dependent/independent mechanisms and enhances ER-stress signalling ${ }^{16}$. Epidemiological studies have shown that maternal virus infection during pregnancy increases the risk of SCZ in the offspring $^{17}$ and animal models based on the prenatal infection hypothesis of SCZ, including prenatal exposure 
to human influenza virus ${ }^{18}$, polyinosinic-polycytidylic acid $\left(\right.$ poly I:C) ${ }^{19}$, lipopolysaccharide (LPS) ${ }^{20}$, turpentine ${ }^{21}$, and interleukin (IL)- $6^{22}$, have been observed to produce behavioural and cognitive dysfunctions. It was suggested that the neuropathological outcomes might be affected by immunological reactions associated with proinflammatory mediators such as IFNs, IL- $1 \beta$, IL- 6 , TNF- $\alpha$, and nitric oxide (NO), which eventually cause neurotoxic effects ${ }^{23}$. Interestingly, our preliminary experiments using poly I:C model mice showed the increase of $M x 1$ gene expression as well $I l 1 b$ and Tnf (Table 4), suggesting that upregulation of $M x 1$ might reflect the dysregulation of immunological reactions associated with the pathophysiology of SCZ.

GART, another promising marker, has phosphoribosylglycinamide formyltransferase, phosphoribosylgly cinamide synthetase, phosphoribosylaminoimidazole synthetase activity, and promotes de novo purine biosynthesis $^{24}$. Extracellular purines can activate astrocytes and microglial cells in response to CNS injury or neurodegeneration and increased levels of purine nucleotides may promote astrocytic hypertrophy ${ }^{25}$. Zhang et al. (2014) ${ }^{26}$ reported that GART is mainly localized in astrocytes and its expression is enhanced after CNS injury and neuroinflammation, which eventually causes astrocyte activation and neural apoptosis. Activated astrocytes release many different types of potentially neurotoxic and proinflammatory mediators, including cytokines and growth factors such as IFNs, IL- $1 \beta$, IL- 6 , TNF- $\alpha$, innate immunity mediators such as LPS and Toll-like receptor (TLR) ligands, neurotransmitters such as glutamate and noradrenalin, purine nucleotides such as adenosine triphosphate (ATP), and reactive oxygen species (ROS) such as $\mathrm{NO}^{27}$. Thus, GART might be involved in the activation of astrocytes and regulate proinflammatory mediator-related immunological reactions ${ }^{26}$. Although poly I:C-treated mice did not show the changes in mRNA level of Gart in both PFC and HIP (Table 4), the most promising molecules might be involved in the pathophysiology of SCZ. Further investigation should be performed to elucidate molecular functions of candidate molecules identified in this study.

\section{Limitation and future research}

A couple of limitations should be considered in the present study. First, although 2D-DIGE technique have improved dynamic range, several issues related to reproducibility due to the properties of proteins (e.g. low abundance, high acidity/basicity, extreme size, or high hydrophobicity). To detect low abundance proteins and maximize discovery rate, we applied high-sensitive spot detection parameters and no multiple testing correction. Although we performed visual inspection of the gel images in 2D-DIGE and Western blotting verification of all candidates from differentially expressed protein spots, appropriate data mining procedures to reduce false discovery should have be considered. Second, the prediction model constructed from eight candidates showed reproducibly high discrimination performance, however, there were inconsistencies of the protein expression change between two sample cohorts of LCLs. The verification of reproducibility using large sample sets should be conducted. Third, it is necessary to consider the utility of different sample types, such as plasma, serum, and peripheral blood mononuclear cells. Different samples analysed using a variety of methodologies have the potential to enhance the understanding of biological processes related to $\mathrm{SCZ}^{28}$.

Differentially expressed protein markers, identified from LCLs, might reflect pathophysiology of disease. Recently available biological resources (e.g. induced pluripotent stem cells and induced microglia-like cells) and genome editing tools (e.g. CRISPER/Cas, TALEN) are undoubtedly powerful technologies for enabling functional analyses of CNS resources such as cell lines or organoids from living donors ${ }^{29,30}$. Future follow-up studies, which investigate molecular mechanisms of identified markers, could provide insight into the pathophysiology of SCZ and potentially provide novel molecular targets and diagnostic/prognostic biomarkers.

\begin{abstract}
Acknowledgements
We sincerely thank the patients and healthy volunteers for their participation in this study. We would like to express our gratitude to Dr. Ryoko Ishihara, Ms. Hiromi Noma, and Ms. Mami Yoshida for technical assistance and discussion, and Ms. Yukari Mitsui for contributions to creating and managing the database. This study was supported by research grants from the Japan Agency for Medical Research and Development (AMED), the Ministry of Education, Culture, Sports, Science and Technology (MEXT) of Japan, the Ministry of Health, Labour and Welfare (MHLW) of Japan, the Japan Society for the Promotion of Science (JSPS), the Japan Science and Technology Agency (JST), Research Group For Schizophrenia, Japan, SENSHIN Medical Research Foundation, Meijo University, the Uehara Memorial Foundation, and GlaxoSmithKline (GSK) Japan; the Grantin-Aid for "Integrated research on neuropsychiatric disorders" carried out under the Strategic Research Program for Brain Sciences from AMED

(JP18dm0107087, JP18dm0207005, JP18dk0307075, JP18dk0307081); the Health and Labour Sciences Research Grants (HLSRG) for Comprehensive Research on Persons with Disabilities from AMED; the Grant-in-Aid for Scientific Research on Innovative Areas, "Glial assembly: a new regulatory machinery of brain function and disorders" from MEXT; the Grant-in-Aid for Scientific Research on Innovative Areas (Comprehensive Brain Science Network) from MEXT; the Grant-in-Aid for Young Scientists B $(25860999,17 \mathrm{~K} 16403)$ from JSPS; the Grant-in-Aid for Scientific Research C (16K08421) from JSPS; the Adaptable \& Seamless Technology Transfer Program through Target-driven R\&D (A-STEP. AS2511400P, AS2621600P) from JST; the Core Research for Evolutional Science and Technology (CREST) from JST; the Academic Frontier Project for Private Universities, Comparative Cognitive Science Institutes, Meijo University; GSK Japan Research Grant 2014.
\end{abstract}

\section{Author details}

'Division of Clinical Sciences and Neuropsychopharmacology, Faculty and Graduate School of Pharmacy, Meijo University, Nagoya 468-8503, Japan. ${ }^{2}$ Department of Neuropsychopharmacology and Hospital Pharmacy, Nagoya University Graduate School of Medicine, Nagoya 466-8550, Japan. ${ }^{3}$ Department of Psychiatry, Nagoya University Graduate School of Medicine, Nagoya 4668550, Japan. ${ }^{4}$ Department of Biostatistics and Bioinformatics, Graduate School of Medicine, University of Tokyo, Tokyo 113-0033, Japan. ${ }^{5}$ Center for Advanced Medicine and Clinical Research, Nagoya University Hospital, Nagoya 466-8550, 
Japan. ${ }^{6}$ Department of Psychiatry, Nagoya University Hospital, Nagoya 4668550, Japan. ${ }^{7}$ Institute for Advanced Research, Nagoya University, Nagoya 4648601, Japan. ${ }^{8}$ Department of Pathology of Mental Diseases, National Institute of Mental Health, National Center of Neurology and Psychiatry, Tokyo 1878553, Japan. ${ }^{9}$ Brain and Mind Research Center, Nagoya University, Nagoya 4668550, Japan

\section{Conflict of interest}

The authors declare that they have no conflict of interest.

\section{Publisher's note}

Springer Nature remains neutral with regard to jurisdictional claims in published maps and institutional affiliations.

Supplementary Information accompanies this paper at (https://doi.org/ 10.1038/s41398-019-0461-2).

Received: 19 December 2018 Revised: 28 January 2019 Accepted: 12 March 2019

Published online: 22 April 2019

\section{References}

1. Owen, M. J., Sawa, A. \& Mortensen, P. B. Schizophrenia. Lancet 388, 86-97 (2016).

2. Penttila, M., Jaaskelainen, E., Hirvonen, N., Isohanni, M. \& Miettunen, J. Duration of untreated psychosis as predictor of long-term outcome in schizophrenia: systematic review and meta-analysis. Br. J. Psychiatry 205, 88-94 (2014).

3. Hayashi-Takagi, A., Vawter, M. P. \& Iwamoto, K. Peripheral biomarkers revisited: integrative profiling of peripheral samples for psychiatric research. Biol. Psychiatry 75, 920-928 (2014).

4. Li, J. Z. et al. Systematic changes in gene expression in postmortem human brains associated with tissue $\mathrm{pH}$ and terminal medical conditions. Hum. Mol. Genet. 13, 609-616 (2004).

5. Rollins, B., Martin, M. V., Morgan, L. \& Vawter, M. P. Analysis of whole genome biomarker expression in blood and brain. Am. J. Med. Genet. B. Neuropsychiatr. Genet. 153B, 919-936 (2010).

6. Sanders, A. R. et al. Transcriptome study of differential expression in schizophrenia. Hum. Mol. Genet. 22, 5001-5014 (2013).

7. Kitchen, R. R., Rozowsky, J. S., Gerstein, M. B. \& Nairn, A. C. Decoding neuroproteomics: integrating the genome, translatome and functional anatomy. Nat. Neurosci. 17, 1491-1499 (2014).

8. Kato, T. et al. Mechanisms of altered $\mathrm{Ca} 2+$ signalling in transformed lymphoblastoid cells from patients with bipolar disorder. Int. J. Neuropsychopharmacol. 6, 379-389 (2003).

9. Hayashi, A. et al. Aberrant endoplasmic reticulum stress response in lymphoblastoid cells from patients with bipolar disorder. Int. J. Neuropsychopharmacol. 12, 33-43 (2009).

10. Yamada, S. et al. Matrix metalloproteinase-3 is a possible mediator of neurodevelopmental impairment due to polyl:C-induced innate immune activation of astrocytes. Brain Behav. Immun. 38, 272-282 (2014).
11. Ibi, D. et al. Neonatal polyl:C treatment in mice results in schizophrenia-like behavioral and neurochemical abnormalities in adulthood. Neurosci. Res. 64 297-305 (2009).

12. Focking, M. et al. Proteomic and genomic evidence implicates the postsynaptic density in schizophrenia. Mol. Psychiatry 20, 424-432 (2015).

13. Hall, J., Trent, S., Thomas, K. L., O'Donovan, M. C. \& Owen, M. J. Genetic risk for schizophrenia: convergence on synaptic pathways involved in plasticity. Biol. Psychiatry 77, 52-58 (2015).

14. Kushima, I. et al. High-resolution copy number variation analysis of schizophrenia in Japan. Mol. Psychiatry 22, 430-440 (2017).

15. Kushima, I. et al. Comparative analyses of copy-number variation in autism spectrum disorder and schizophrenia reveal etiological overlap and biological insights. Cell Rep. 24, 2838-2856 (2018).

16. Numajiri Haruki, A., Naito, T., Nishie, T., Saito, S. \& Nagata, K. Interferon-inducible antiviral protein MxA enhances cell death triggered by endoplasmic reticulum stress. J. Interferon Cytokine Res. 31, 847-856 (2011).

17. Meyer, U. Prenatal poly(i:C) exposure and other developmental immune activation models in rodent systems. Biol. Psychiatry. 75, 307-315 (2014).

18. Kneeland, R. E. \& Fatemi, S. H. Viral infection, inflammation and schizophrenia. Prog. Neuropsychopharmacol. Biol. Psychiatry 42, 35-48 (2013).

19. Meyer, U. \& Feldon, J. To poly(l:C) or not to poly(l:C): advancing preclinical schizophrenia research through the use of prenatal immune activation models. Neuropharmacology 62, 1308-1321 (2012).

20. Willette, A. A. et al. Brain enlargement and increased behavioral and cytokine reactivity in infant monkeys following acute prenatal endotoxemia. Behav. Brain. Res. 219, 108-115 (2011).

21. Aguilar-Valles, A. \& Luheshi, G. N. Alterations in cognitive function and behavioral response to amphetamine induced by prenatal inflammation are dependent on the stage of pregnancy. Psychoneuroendocrinology. 36, 634-648 (2011).

22. Smith, S. E., Li, J., Garbett, K., Mirnics, K. \& Patterson, P. H. Maternal immune activation alters fetal brain development through interleukin-6. J. Neurosci. 27, 10695-10702 (2007).

23. Fritz-French, C. \& Tyor, W. Interferon-alpha (IFNalpha) neurotoxicity. Cytokine Growth Factor Rev. 23, 7-14 (2012).

24. Aimi, J., Qiu, H., Williams, J., Zalkin, H. \& Dixon, J. E. De novo purine nucleotide biosynthesis: cloning of human and avian cDNAs encoding the trifunctional glycinamide ribonucleotide synthetase-aminoimidazole ribonucleotide synthetase-glycinamide ribonucleotide transformylase by functional complementation in E. coli. Nucleic Acids Res. 18, 6665-6672 (1990).

25. Banerjee, D. \& Nandagopal, K. Potential interaction between the GARS-AIRSGART Gene and CP2/LBP-1C/LSF transcription factor in Down syndromerelated Alzheimer disease. Cell. Mol. Neurobiol. 27, 1117-1126 (2007).

26. Zhang, D. et al. GART expression in rat spinal cord after injury and its role in inflammation. Brain Res. 1564, 41-51 (2014).

27. Owens, T., Khorooshi, R., Wlodarczyk, A. \& Asgari, N. Interferons in the central nervous system: a few instruments play many tunes. Glia 62, 339-355 (2014).

28. Herberth, M. et al. Peripheral profiling analysis for bipolar disorder reveals markers associated with reduced cell survival. Proteomics. 11, 94-105 (2011).

29. Mariani, J. et al. FOXG1-dependent dysregulation of GABA/Glutamate neuron differentiation in autism spectrum disorders. Cell. 162, 375-390 (2015).

30. Arioka, Y., Kushima, I., Kubo, H., Mori, D. \& Ozaki, N. Induced pluripotent stem cells derived from a schizophrenia patient with ASTN2 deletion. Stem Cell Res 30, 81-84 (2018). 